\title{
GLOBAL COMPUTING
}

\author{
Matthew N. O. Sadiku, Kelechi G. Eze, and Sarhan M. Musa
}

\author{
Roy G. Perry College of Engineering \\ Prairie View A\&M University \\ Prairie View, TX 77446
}

\begin{abstract}
Global computing refers to an approach that relies on a distribution of data over a large amount of data sources. Such a computing system gathers thousands of resources to enable parallel computing applications. Global computing is a computing approach for computers distributed over the world. Its goal is to harness the Internet's vast computational capacity for large parallel application. This paper provides a brief introduction to global computing.
\end{abstract}

Key Words: Global Computing, World-Wide Computing, Global Computation, Wide-Area Computation, Wide-Area Network Programming, Internet Programming.

\section{INTRODUCTION}

The Internet has been expanding exponentially in recent years. Its ever-growing popularity and the wide-acceptance of the World Wide Web have motivated numerous research works leading to various computing schemes. The Internet is showing the potential to form a supercomputing resource out of computers connected to it.

Computing is increasingly characterised by the global scale of applications. Global computing has the idea of harnessing computational power of networked computers.

Global computing (also known as wide-area computation, wide-area network programming or Internet programming) is a discipline aiming to harvest the idle time of Internet connected computers to run very large distributed applications. It allows users to access and share computational resources including hardware, software, and data published by others across a wide area network (WAN). Global computing represents a paradigm shift from storing data in monolithic data management systems towards seeing it distributed over a large number of small-scale, mobile, data carrying devices.

The global computing system may be regarded as the natural evolution of the Internet.

It harnesses global resources (CPU time, memory, disk storage) and make them available to every user on the Internet. In such a global computing environment, the mobile devices contain various kinds of data: content data, profile data, and essential metadata [1]. Global computing is related to grid computing, cluster computing, and Internet computing.

\section{CONCEPT OF GLOBAL COMPUTING}

The key notion of global computing is harvesting the idle time of Internet connected computers and transparently employing them to solve large-scale problems. All the computing power is provided by volunteer computers, which are spread around the globe. Thus global computing extends the cycle stealing model across the Internet. A typical architecture of global computing systems is shown in Figure 1 [2].

The main feature of a global computer is its geographical distribution since it interconnects various devices such as the clusters, PCs, PDAs, handsets, and mobile phones which may be widely scattered geographically. Global computing (WAN programming) distinguishes itself from local area network computing (LAN computing).

The main components of a global computing system include the following [3]:

- Communication Performance: Global computing requires large amounts of data to be shipped over the network. Computation will be typically performed on high-performance supercomputing servers such as vector supercomputers.

- $\quad$ Sharing by Multiple Clients: Computing servers will be shared by arbitrary multiple clients on the network. Computation task is shared among many components for the clients to execute. Since we rely on existing supercomputers, it is not apparent whether their operating systems could handle multiple computational requests from clients. 
- Remote Library Design and Reuse: When executing remote libraries registered on the computing servers, there is an option as to whether to distribute the computing resources amongst different client requests in a task parallel manner, or to allocate all the processors to each client task.

These components are essentially for Ninf which is currently based on the client-server model, but they apply to other global computing systems.

\section{PROPOSED GLOBAL COMPUTERS}

Several high performance global computing systems have been proposed worldwide. Due to limited space, we will not describe them in detail here. They are summarized as follows [4]:

- USA: Globus, Legion, WebFlow, NetSolve, and NASA IPG.

- Asia/Japan: Ninf and Bricks

- Australia: Nimrod/G and DISCWorld.

- Europe: UNICORE, CERN Data Grid, MOL, Globe, DAS, MetaMPI.

Others include RCS, CONDOR, Javelin++, Bayaniha, Grid, XtremWeb, SuperWeb SETI@home, GLOBAL GAMMA, ATLAS, and DB-Globe. Each of these proposed systems intends to effectively achieve high performance with some efficient scheduling scheme. Following the success stories of the proposed global computers, global computing is receiving more and more attention from industry and academia.

\section{BENEFITS AND CHALLENGES}

Global computing provides some benefits to society, but their adverse effects should not be overlooked. An important feature of global computing system is location transparency; information can be obtained irrespective of time or location. Global computing is beneficial for a user who is looking for some extra computational power to run parallel supercomputing applications. Corporations can use global computers to run large parallel and distributed applications with performance far superior to today's supercomputers, at a fraction of the cost [5]. Every large enterprise will soon have its own internal global computer connecting its geographically distributed resources.

A major challenge will how multiple, heterogeneous global computers can interact effectively and securely. A model of security is crucial for mobile computation. Java deals with security and multiple global computers [6]. Such a programming language comes equipped with a basic model that also encompasses security features. Java-based global parallel computing makes programming easy. Javelin is another Java-based infrastructure for global computing [7].

Although global computations are expected to tolerate certain low rates of faults, massive attacks can occur when an error rate is larger than tolerable. Such massive attacks may be due to distributed denial of service.

With the current Internet lacking sufficient bandwidth communication would become the bottleneck. In LAN environment, computing server performance dictates overall performance, whereas in WAN setting limitation of communication throughput is more significant. It is difficult to perform large-scale benchmarks on the Internet with reproducible results.

\section{CONCLUSION}

Global computing is a method for computing in a wide area network such as the Internet. It is an approach that can be used for computing on the Internet. Global computing systems are proposed to harness Internet-connected resources at a global scale. They make use of volunteered computing power to obtain computing power without investing in hardware and electricity. They require at least an order of magnitude more computers than conventional computation. More information about global computing can be found in books in $[8,9]$.

\section{REFERENCES}

[1] D. Pfoser, E. Pitoura, and N. Tryfona, "Metadata modeling in a global computing environment," Proceedings of the 10 ${ }^{\text {th }}$ ACM International Symposium on Advances in Geographic Information Systems, 2002, pp. 68-73.

[2] K. Aida et al., "Performance evaluation model for scheduling in global computing systems," The International Journal of High Performance Computing Applications, vol. 14, no. 3, Fall 2000, pp. 268-279.

[3] A. Takefus et al., "Multi-client LAN/WAN performance analysis of Ninf: a high-performance global computing system," Proceedings of the 1997 ACM/IEEE Conference on Supercomputing, San Jose, CA., Nov. 1997, pp. 1-23.

[4] M. Baker, R. Buyya, and D. Laforenza, "The Grid: International efforts in global computing," https://pdfs.semanticscholar.org/333b/8725e7c7b06ed0d55ab64cfe792812ff687f.pdf

[5] A. D. Alexandrov et al., "SuperWeb: Research issues in Java-based global computing," Concurrency: Practice and Experience, vol.9, no. 6, June 1997, pp. 535-553. 
[6] L. Carde, “Global computation,” ACM Computing Surveys, vol. 28, no. 4, Dec, 1996, pp. 66-68.

[7] M. O. Neary, "Javelin++: Scalability issues in global computing," Concurrency: Practice and Experience, vol. 12, 2000, pp. $727-753$.

[8] R. Home, Embracing Global Computing in Emerging Economies. Springer, 2015.

[9] M. Erby, Trustworthy Global Computing. Springer, 2018.

\section{ABOUT THE AUTHORS}

Matthew N.O. Sadiku is a professor in the Department of Electrical and Computer Engineering at Prairie View A\&M University, Prairie View, Texas. He is the author of several books and papers. His areas of research interest include computational electromagnetics and computer networks. He is a fellow of IEEE.

Kelechi G. Eze is a doctoral student at Prairie View A\&M University, Texas. He is a student member of IEEE. His research interests include Internet of things security, data security and privacy, blockchain technology, wireless sensor networks, and machine learning.

Sarhan M. Musa is a professor in the Department of Engineering Technology at Prairie View A\&M University, Texas. He has been the director of Prairie View Networking Academy, Texas, since 2004. He is an LTD Sprint and Boeing Welliver Fellow.

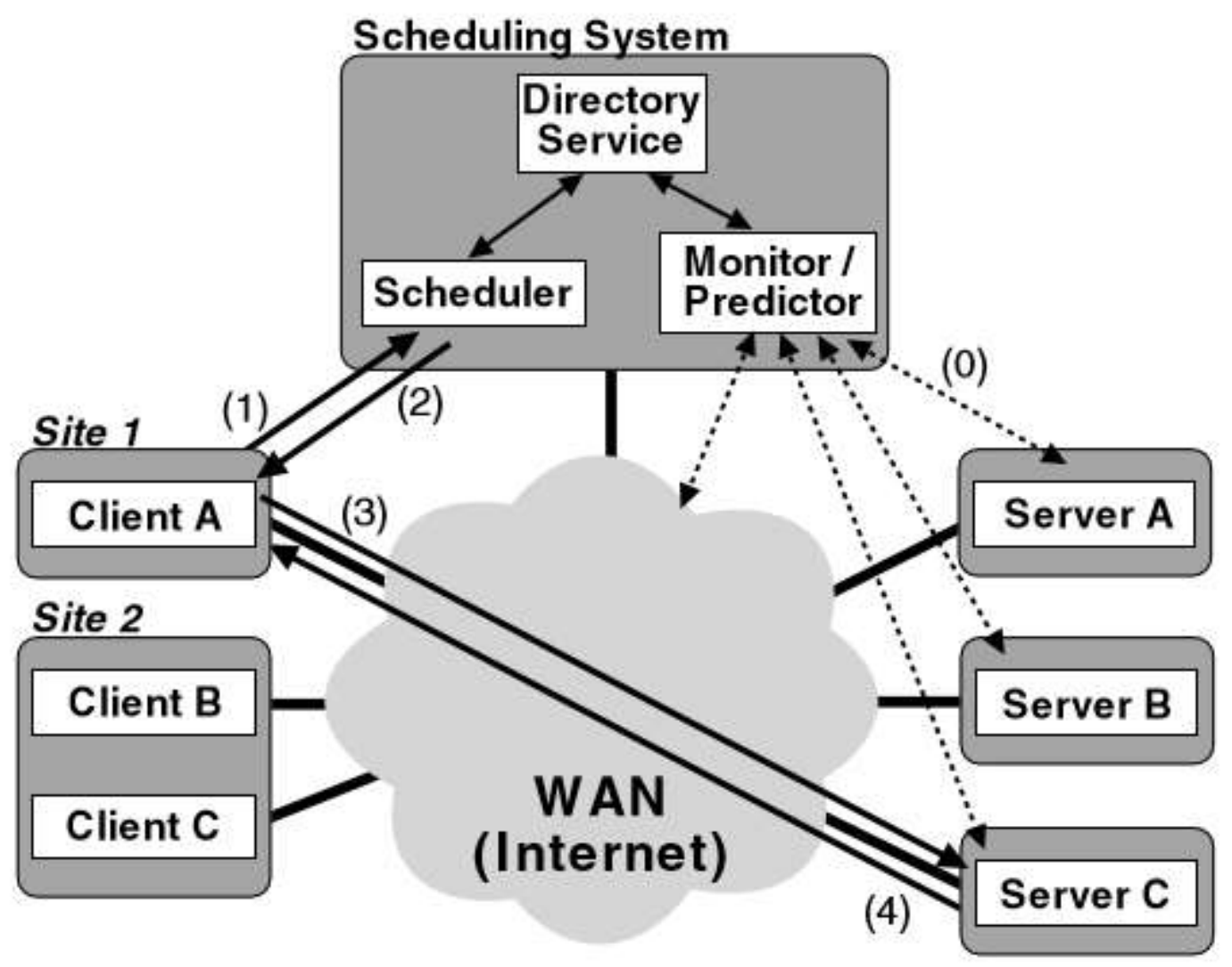

Figure 1 A typical architecture of global computing systems [2]. 